\title{
3-D MICROMACHINED SPHERICAL SHELL RESONATORS WITH INTEGRATED ELECTROMAGNETIC AND ELECTROSTATIC TRANSDUCERS
}

\author{
S.A. Zotov, I.P. Prikhodko, A.A. Trusov*, A.M. Shkel
}

MicroSystems Laboratory, Mechanical and Aerospace Engineering, University of California, Irvine, CA, USA

\begin{abstract}
This paper presents a new paradigm for design and fabrication of 3-D spherical shell resonators. The approach uses pressure and surface tension driven plastic deformation (glassblowing) on a wafer scale as a mechanism for creation of inherently smooth and symmetric 3-D resonant structures with integrated electrodes. Feasibility of the new approach was demonstrated by fabrication and characterization of Pyrex glass spherical shell resonators with millimeter scale diameter and average thickness of 10-20 $\mu \mathrm{m}$. Integrated 3-D metal electrodes were used to actuate the two dynamically balanced 4-node wineglass modes. For $1 \mathrm{MHz}$ glassblown resonators, the relative frequency mismatch $\Delta f / f$ between the two degenerate wineglass modes was measured as $0.63 \%$ without any trimming or tuning. The intrinsic manufacturing symmetry and high aspect ratio transducer architectures of the proposed technology may enable new classes of high performance 3-D MEMS for communications and navigation applications.
\end{abstract}

\section{INTRODUCTION}

Miniature resonators are required for a range of applications, including signal processing, timing, frequency control, and inertial sensing [1]. A wide array of surface and bulk fabrication technologies for silicon MEMS resonators has been developed over the past decades with several commercial silicon resonators currently entering the consumer electronics market. Conventional planar technologies for MEMS resonator fabrication rely on photolithography and silicon DRIE which provide relative fabrication tolerances on the order of $1 \%$. The fabrication imperfections, surface roughness, DRIE induced scalloping and footing, as well as aspect ratio limitations present challenges in device symmetry, frequency specification and matching, quality factor maximization, and further device miniaturization without performance sacrifice. These factors motivate the investigation of alternative, non-planar architectures and technologies for resonant MEMS with simultaneously increased symmetry and aspect ratios.

This paper explores the hypothesis that plastic deformation driven by surface tension and pressure, or glassblowing [2], may serve as an enabling mechanism for wafer-scale fabrication of highly smooth and symmetric 3-D spherical shell resonators. The proposed micromachining approach consists of 1) bonding a Pyrex glass wafer on a silicon substrate with pre-etched cavities 2) patterning of flat metal features on the silicon-on-glass substrate 3) heating the wafer stack above the softening point of glass. The induced plastic deformation of the metal-on-glass stack creates 3-D spherical shells with integrated metal electrodes, Fig. 1 and 2.

Operation of continuous, dynamically balanced wineglass modes in 3-D axisymmetric shells provides increased robustness to fabrication imperfections. In conventional planar mass-spring type resonators, linear sensitivity of the natural frequency $f$ to fabrication imperfections is governed by $\Delta f / f \propto(3 / 2) \Delta w / w$, where $w$ is the nominal suspension beam width and $\Delta w$ is the associated fabrication tolerance. In contrast, variation $\Delta w$ of the nominal shell thickness $w$ appears only as a second order term in the relative frequency mismatch $\Delta f / f \propto(3 / 4)(\Delta w / w)^{2}$ for a spherical shell resonator operated in a balanced wineglass mode [3]. This paper presents the glassblowing approach to 3-D shell resonator fabrication and reports experimental characterization.

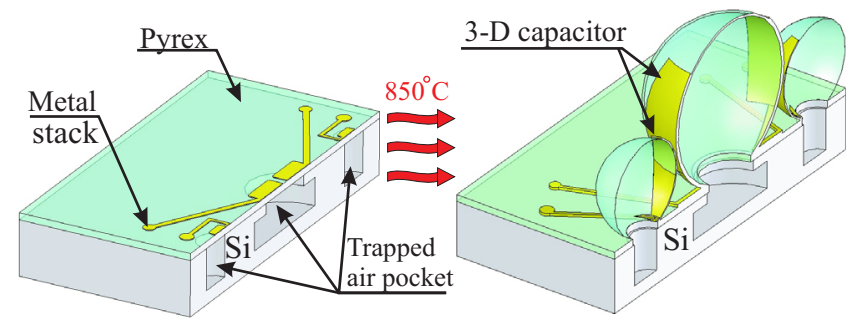

(a) Before glassblowing

(b) After glassblowing

Figure 1: Conceptual schematic of 3-D spherical shell resonator fabrication using wafer-scale metal-on-glass stack glassblowing.

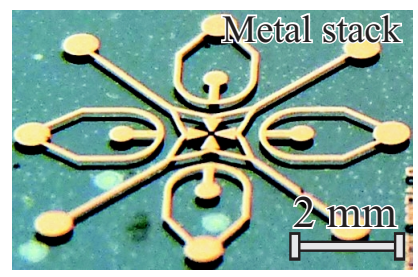

(a) Before glassblowing

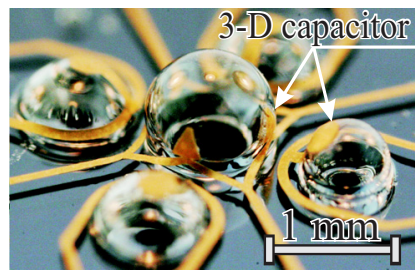

(b) After glassblowing
Figure 2: Photographs of fabricated 3-D spherical shell resonators with integrated electrostatic transducers.

\section{GLASS-BLOWN SHELL AS VIBRATORY ELEMENT}

Fabrication of 3-D resonant shells relies on wafer-scale glassblowing, which was previously developed for creation of extremely smooth and symmetric spherical cells for chip-scale atomic devices [4]. The process starts by hermitically bonding a $100 \mu \mathrm{m}$ thick Corning Pyrex 7740 glass wafer to a silicon wafer with an array of $0.5 \mathrm{~mm}$ diameter cylindrical cavities etched $0.8 \mathrm{~mm}$ deep, Fig. 1(a). The wafer stack is then heated above the $850{ }^{\circ} \mathrm{C}$ softening point of Pyrex glass. Above this temperature, the viscosity of glass reduces, and the expansion of the air trapped inside the pre-etched silicon cavities causes the flat glass to be blown into spherical shapes, Fig. 1(b).

\section{Surface Quality of Glass-Blown Spheres}

Surface roughness is a known performance limiting effect in microscale resonators. Development of surface tension driven glassblowing process is expected to provide surface roughness improvement compared to the conventional fabrication processes based on etching. To investigate this hypothesis experimentally, several glass-blown shells were characterized using an Agilent Nano R2 Atomic Force Microscope (AFM). The initial average surface roughness of the Pyrex 7740 wafer before glassblowing was measured as $0.48 \mathrm{~nm}$ over a $(5.2 \mu \mathrm{m})^{2}$ area. The average surface roughness after the fabrication was measured as $0.85 \mathrm{~nm}$ on the outer shell surface. This translates into the overall $1 \mathrm{ppm}$ figure of merit relative to the $0.9 \mathrm{~mm}$ diameter of the shell, Fig. 3 . The uncertainty of both AFM measurements was on the order of $\pm 0.2 \mathrm{~nm}$. The final shell roughness is comparable to the initial glass roughness before the fabrication. Further reduction in shell roughness can be achieved by controlling the level of impurities and contaminants in the nitrogen atmosphere of the glassblowing furnace.

To compare the glassblowing technology with conventional surface micromachining, the same set of measurements was 


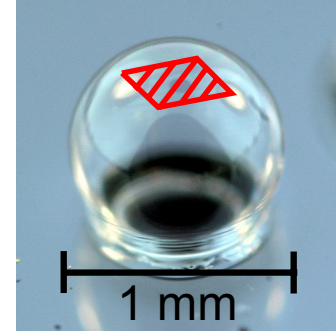

(a) $0.9 \mathrm{~mm}$ glass-blown shell $5.2 \times 5.2 \mu$ m measured area

Figure 3: Glass shell surface roughness measured with an atomic force microscope. Relative roughness measurement is 1 ppm.

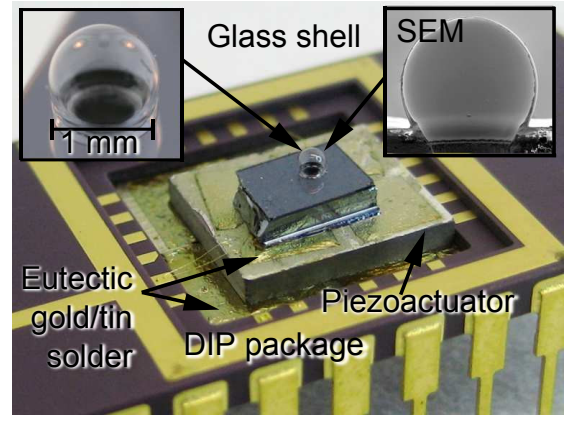

Figure 4: Photograph of a packaged glass-blown shell bonded to a piezoelectric actuator for structural mode characterization.

performed on a sidewall of a silicon resonator defined by DRIE using Unaxis Versaline VL-7339. Surface defects were measured as $17 \mathrm{~nm}$ over the same area, equivalent to $167 \mathrm{ppm}$ roughness relative to the $100 \mu \mathrm{m}$ beam thickness. This comparison demonstrates the potential of the glassblowing technology to produce high aspect ratio 3-D resonators with very low surface roughness.

\section{Non-Contact Characterization of Resonant Modes}

Inherent fabrication symmetry and measured low surface roughness suggest that glass-blown spheres can serve as high quality resonant structures with superior degree of isotropy. This section investigates the resonant properties of fabricated spheres using non-contact characterization methods facilitated by a Laser Doppler Vibrometer (LDV). For the experiment, a 3-D shell die was eutectically bonded to a piezoelectric (PZT) plate actuator and packaged using a ceramic DIP package, Fig. 4. The PZT was chosen for the preliminary experiments for simple excitation of multiple order vibratory modes in spatial 3-D structures. A Polytec OFV5000 single-point LDV was used to directly measure velocities of points on the vibrating shell surface in real time.

Finite Element Modeling (FEM) of the glass-blown shell with a $0.9 \mathrm{~mm}$ diameter and a $10 \mu \mathrm{m}$ average thickness predicted that dynamically balanced 4-node wineglass modes occur at the resonant frequencies of $1.271 \mathrm{MHz} \pm 0.3 \%$. For the experimental characterization, the packaged glass-blown shell was mounted on a rotary stage in focus of the LDV, Fig. 5. The PZT substrate was driven with $1 \mathrm{~V} \mathrm{AC}$ at frequencies ranging from 1.25 to $1.29 \mathrm{MHz}$. In order to measure velocity as a function of the angular position on the shell (longitude), a scan was performed by rotating the packaged device with respect to the stationary LDV beam in $15^{\circ}$ increments of longitude. Fig. 6 shows frequency responses of the glass-blown shell at several locations in the equatorial plane. The measurement reveals a $0.63 \%$ relative frequency mismatch $\Delta f / f$ between the two theoretically degenerate wineglass modes.

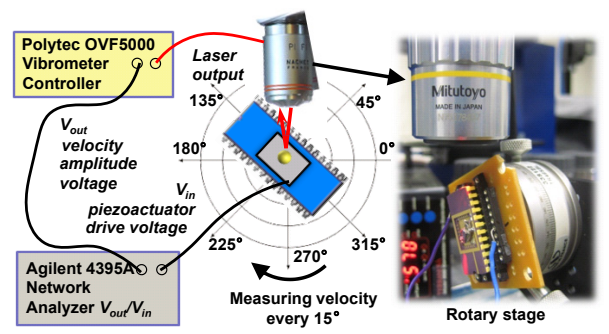

Figure 5: Schematic of the experimental testbed for dynamic characterization of glass-blown shells as mechanical elements.

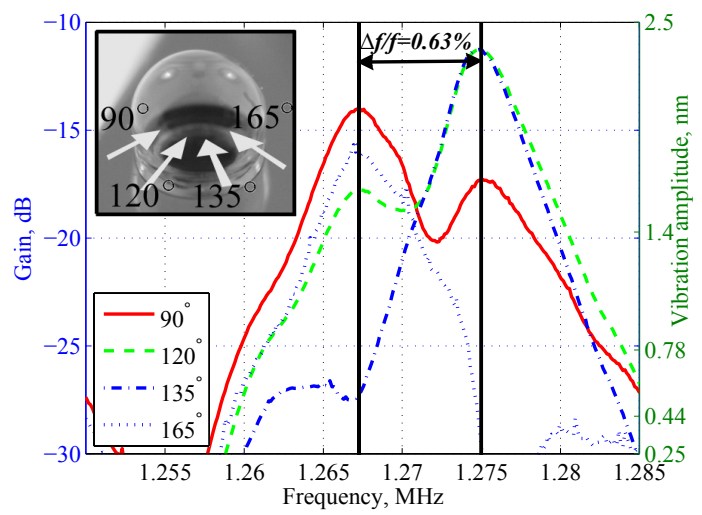

Figure 6: Measured frequency responses corresponding to the 4-node wineglass modes, revealing mismatch $\Delta f / f$ of only $0.63 \%$.

Next, we analyzed the mode-shapes corresponding to the two resonant frequencies and compared them to the theoretical 4-node wineglass velocity distributions. Velocity amplitudes were measured at 24 equally spaced points along the shell equator and plotted in polar coordinates, Fig. 7. As expected from FEM, the elastic wave patterns exhibit 4 points with zero displacement, which is a characteristic property of a 4-node wineglass mode [1]. The relative spatial orientation between the two 4-node wineglass velocity patterns is defined by the principal axes of elasticity. For a fixed latitude $\theta$ cross section, the radial displacement $r$ of an order $m$ vibratory mode is a function of time $t$ and longitude angle $\varphi$ [5]:

$$
r(\varphi)=P_{n}^{m}(\cos \theta) \sin \left(m \varphi+m \varphi_{0}\right) \cos (\omega t)
$$

where $P_{n}^{m}(\cdot)$ is the associated Legendre function and $\omega$ is the vibration frequency. The experimental velocity amplitude maps measured at the equator for $m=2$ mode were fitted to this analytical model, revealing $\varphi_{0}=27^{\circ}$ angle between the principal axes of elasticity, Fig. 7. The non-contact characterization using off-chip actuation revealed isotropy and principal axis alignment on par with the state-of-the-art planar silicon MEMS [1], calling for the development of integrated on-chip transducers.

\section{TRANSDUCER INTEGRATION}

Integration of electromagnetic and electrostatic transducers with the glass-blown spherical resonators requires definition of conductive traces and electrodes on the curved 3-D surfaces of the spherical structures. Conventional methods such as line-of-sight metal deposition using a shadow mask are not practical due to the aspect ratio limitations.

\section{3-D Metal Fabrication}

Instead of patterning metal conductors on the curved surface of already glass-blown spherical structures, the explored fabrication approach relied on definition of metal features prior to the glassblowing step, Fig. 8(a). The flat metal features are deposited on top of the Pyrex substrate using, for instance, 


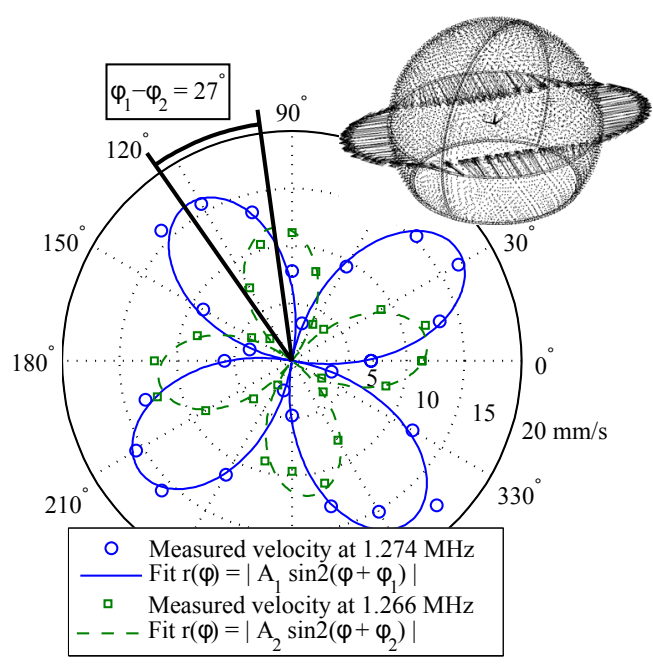

Figure 7: Measured velocity distribution along the equator of a spherical shell excited into 4-node wineglass modes by PZT.

conventional lift off procedure. When the bonded Pyrex-Si wafer stack with patterned metal structures is heated above the $850{ }^{\circ} \mathrm{C}$ softening point of glass, spherical glass structures with integrated 3-D metal electrodes and traces are created, Fig. 8(b).

The main practical challenge of the proposed technique was devising a metal stack that is ductile enough to plastically deform together with the glass substrate during the glassblowing step. At the same time, the metal conductor should be resistant to degradation during the high-temperature treatment. The initial approach of using a stack of $0.5 \mu \mathrm{m} \mathrm{Au}$ and $0.05 \mu \mathrm{m}$ Cr proved intricate due to the partial segmentation of the continuous traces during the plastic deformation phase of the glassblowing step. This limitation was overcome after multiple design iterations by introducing an intermediate $0.8 \mu \mathrm{m}$ thick layer of ductile $\mathrm{Cu}$ between the $\mathrm{Cr}$ adhesion layer and the $\mathrm{Au}$ corrosion resistant top layer. Fig. 1(b) and 8(b) show fabricated samples containing glassblown spherical shells with integrated 3-D metal electrodes and traces, formed from a flat Pyrex glass wafer with patterned $\mathrm{Cr}-\mathrm{Cu}-$ Au metal stack.

\section{Design of Integrated Transducers}

Successful fabrication of the patterned metal features on the surface of glass-blown spherical structures allowed for the integration of electromagnetic and electrostatic transducers. The explored architecture for on-chip electromagnetic transducers utilizes 3-D metal traces on the surface of a glass-blown shell, Fig. 8. The metal trace was patterned as a folded coil to account for the stretching of the metal trace during the plastic deformation of the glass shell. The final 3-D geometry of the shell and metal electrodes was defined by the glassblowing step, which created a $20 \mu \mathrm{m}$ wide 3 -D metal trace across a spherical resonant shell of $1.3 \mathrm{~mm}$ diameter, Fig. 8(b).

For the generation of magnetic field a pair of millimeter-scale 0.5 Tesla permanent magnets was bonded to the die on opposite sides of the glass-blown shell, Fig. 9. When a current is passed through the metal trace on the spherical shell, a distributed Lorentz force is generated which is used to excite mechanical response of the spherical shell. While electromagnetic actuation is commonly used in micro- and nano-scale resonators [6], its drawback is the demanding requirement for strong magnetic fields (on the order of 1 Tesla), which are usually achieved by using external permanent magnets or superconductive coils.

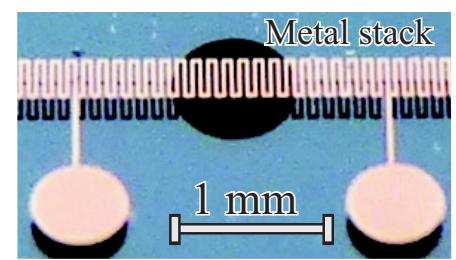

(a) Before glassblowing

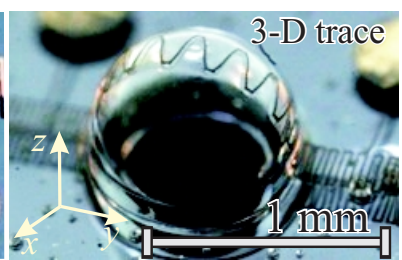

(b) After glassblowing
Figure 8: Photographs of a continuous $20 \mu \mathrm{m}$ wide, $130 \Omega$ metal trace fabricated on a glass-blown spherical shell structure.

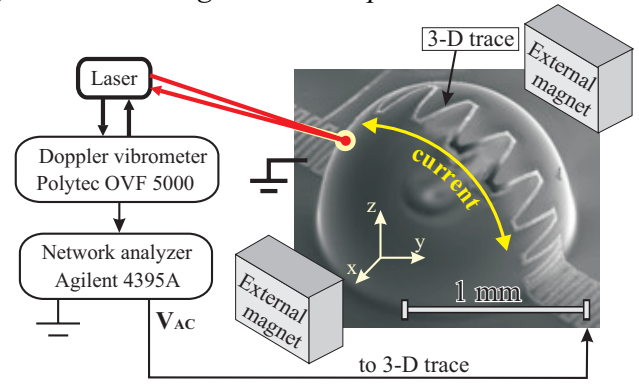

Figure 9: Schematic of the experimental testbed for on-chip electromagnetic excitation of glass-blown resonant shells.

The 3-D metal fabrication technology was also used to implement electrostatic transducers integrated with glass-blown spherical resonators. The idea behind the design of the 3-D capacitors for electrostatic transduction is to form a large-area, high aspect ratio capacitor between the resonant shell and stationary electrodes supported by satellite spherical structures, Fig. 1 and 2. The diameter of the implemented central resonant shell was approximately $1.3 \mathrm{~mm}$, while the satellite smaller shells were of $0.85 \mathrm{~mm}$ in diameter.

\section{ON-CHIP TRANSDUCER CHARACTERIZATION}

Feasibility of the 3-D spherical glass-blown resonators with integrated transduction was experimentally demonstrated by driving the central resonant shell into mechanical resonance.

\section{Electromagnetic Actuation}

Operation of the 3-D electromagnetic transducers was characterized experimentally using a spherical shell with integrated $20 \mu \mathrm{m}$ wide, $130 \Omega$ metal trace running across the shell, Fig. 8. An AC actuation current of $0.2 \mathrm{~mA}$ was passed through the metal trace in presence of a 0.5 Tesla magnetic field generated by the two millimeter scale permanent magnets, Fig. 9. A Lorentz force on the order of $0.5 \mu \mathrm{N}$ was generated to actuate vibrations of the glassblown shell. The mechanical response of the shell was measured using the LDV. Frequency response characterization revealed a $687 \mathrm{kHz}$ resonance corresponding to a rocking mode of shell vibrations, Fig. 10. While this experiment demonstrated operational 3-D electromagnetic resonators with an integrated actuator, requirement for relatively high currents and magnetic fields motivated the investigation of alternative transduction approaches.

\section{Electrostatic Actuation}

Performance of the integrated 3-D electrostatic transducers was characterized using a fabricated prototype with 4 discrete capacitors formed between the central resonant shell and the smaller satellite structures, Fig. 1(b). Unlike the previous electromagnetic architecture, the electrostatic design ensures selective excitation of the 4-node wineglass modes which provides inherent rejection of common mode accelerations and has potential 


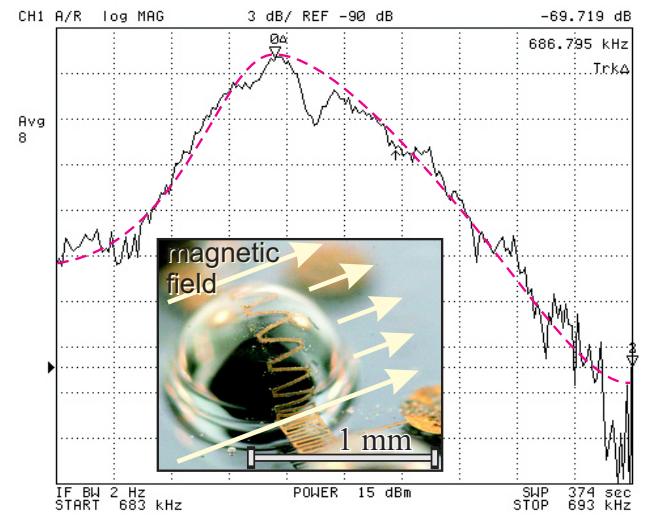

Figure 10: Measured frequency response of a glass-blown shell actuated with an on-chip 3-D magnetic transducer.

for high quality factor and low phase noise operation [1]. To excite vibrations of the central shells, a combination of DC and AC voltages was applied across the driving capacitors, Fig. 11. Mechanical response of the electrostatically excited resonator was characterized by measuring velocities at 36 equally spaced locations along the shell equator using the LDV. A 4-node wineglass mode of vibration was identified at $943.7 \mathrm{kHz}$. This resonant frequency differs from $1.27 \mathrm{MHz}$ previously observed using PZT due to the increased diameter of the shell with integrated metal ( $1.3 \mathrm{~mm}$ compared to $0.9 \mathrm{~mm}$ ).

Velocity amplitude data for the shell equator vibrating at $943.7 \mathrm{kHz}$ was collected and plotted in polar coordinates to analyze the mode-shape in more detail, Fig. 12. The measurement data shows a characteristic pattern of a wineglass mode with 4 nodes and 4 points of maximum velocity amplitude. Analysis of the velocity phase data revealed 4 regions of $180^{\circ}$ alternating phase, confirming the 4-node wineglass mode. The experiments demonstrate feasibility of integrated 3-D capacitors for transduction of dynamically balanced wineglass modes in glassblown spherical shell resonators.

\section{CONCLUSIONS}

A new technology for fabrication of 3-D spherical shell MEMS resonators with integrated electromagnetic and electrostatic transduction was developed and experimentally demonstrated. The fabrication approach takes advantage of plastic deformation driven by pressure and surface tension forces (glassblowing) to create smooth and symmetric, high aspect ratio 3-D structures. The axisymmetric 3-D shell architectures demonstrate surface roughness on the order of $1 \mathrm{ppm}$ and provide inherent robustness to fabrication imperfections. Without any trimming or tuning of the natural frequencies, a $1 \mathrm{MHz}$ shell resonator demonstrated a $0.63 \%$ frequency mismatch between two degenerate wineglass modes, on par with the state-of-the-art planar silicon MEMS. Even though the presented results are the initial steps toward mature dynamic 3-D MEMS, we believe the approach may lead to high precision devices with high aspect ratio architectures, efficient low noise transduction, increased stability, and reduced energy dissipation. The approach may lead to a new class of inertial instruments and truly exploiting the physics of 3-D elastic waves on micro-scale.

\section{ACKNOWLEDGEMENTS}

This work was supported by the National Science Foundation grant CMMI-0928999. Design, modeling, and characterization was performed in the UCI Microsystems Laboratory. The authors

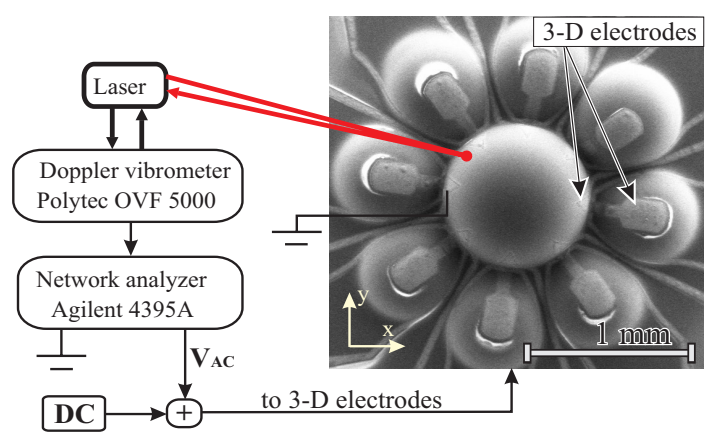

Figure 11: Schematic of the experimental testbed for on-chip electrostatic excitation of glass-blown resonant shells.

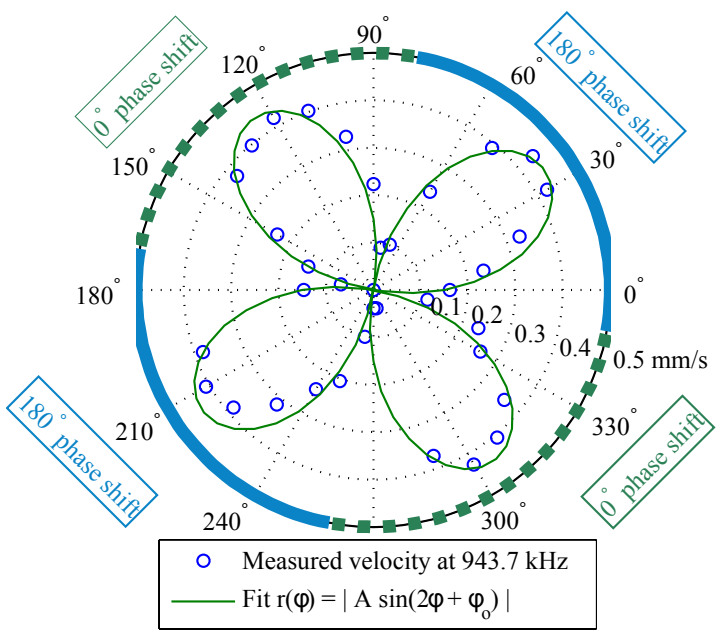

Figure 12: Measured velocity amplitude and phase distribution along the equator of a spherical shell vibrating in a 4-node wineglass mode excited by on-chip capacitive transducers.

would like to acknowledge the UCI Integrated Nanosystems Research Facility and Materials Characterization Center.

\section{REFERENCES}

[1] C. Nguyen, "MEMS Technology for Timing and Frequency Control," IEEE Trans. Ultrasonics, Ferroelectrics and Frequency Control, vol. 54, no. 2, pp. 251-270, Feb. 2007.

[2] E. J. Eklund, A. M. Shkel, "Glass Blowing on a Wafer Level,” IEEE JMEMS, vol. 16, no. 2, pp. 232-239, 2007.

[3] E. J. Loper, D. D. Lynch, K. M. Stevenson, "Projected Performance of Smaller Hemispherical Resonator Gyros," Proc. Position Location and Navigation Symposium, 61-64, 1986.

[4] E. J. Eklund, A. M. Shkel, S. Knappe, E. Donley, J. Kitching, "Glass-blown Spherical Microcells for Chip-Scale Atomic Devices," Sensors and Actuators A: Physical, Vol. 143, Issue 1, pp. 175-180, 2008.

[5] F. I. Niordson, "Free Vibrations of Thin Elastic Spherical Shells,” Int. J. Solids Structures, vol. 20, pp. 667-687, 1984.

[6] A. N. Cleland, M. L. Roukes, "Fabrication of High Frequency Nanometer Scale Mechanical Resonators from Bulk Si Crystals," Appl. Phys. Lett., vol. 69, no. 18, pp. 2653-2655, 1996.

\section{CONTACT}

* A.A. Trusov, tel: +1-949-824-6314; atrusov@uci.edu. 